\title{
Salivary biomarkers of HPA axis and autonomic activity in adults with intellectual disability with and without stereotyped and self-injurious behavior disorders
}

\author{
Frank J. Symons • Jason J. Wolff • Laura S. Stone • \\ Tony K. Y. Lim • James W. Bodfish
}

Received: 9 September 2010 / Accepted: 25 March 2011 /Published online: 12 April 2011

(C) Springer Science+Business Media, LLC 2011

\begin{abstract}
Salivary levels of biomarkers for the hypothalamicpituitary-adrenal axis (HPA; cortisol) and sympatho-adrenomedullary system (SAM; $\alpha$-amylase) were measured in 51 adults (57\% male) with neurodevelopmental disorders associated with intellectual disability (i.e., mental retardation) and chronic self-injurious behavior (SIB) and compared with matched controls without SIB. Cortisol levels differed significantly $(p<0.01)$ between the SIB and control group (SIB $>$ control). Within-group analyses showed significant differences $(p<0.05)$ in levels of salivary $\alpha$-amylase between individuals with SIB and those with SIB meeting criteria for stereotyped movement disorder (SMD; SIB + SMD $>$ SIB). Salivary $\alpha$ amylase was significantly correlated with frequency of stereotypy among the SIB group $(r=0.36, p<0.05)$. These preliminary findings warrant further exploration into the role of the SAM system in the pathophysiology of SIB and related
\end{abstract}

The preparation of this report was supported, in part, by NICHD Grant No. 44763 and 47201 to the University of Minnesota. We are grateful to Jamie Clary for assistance with saliva collection. Our sincere thanks go to a committed group of parents and staff for their support.

F. J. Symons $(\bowtie)$

Department of Educational Psychology, Center for

Neurobehavioral Development, University of Minnesota,

Education Science Building, 56 River Road,

Minneapolis, MN 55455, USA

e-mail: symon007@umn.edu

J. J. Wolff $\cdot$ J. W. Bodfish

Carolina Institute for Developmental Disabilities,

University of North Carolina at Chapel Hill,

200 East Cameron Avenue,

Chapell Hill, NC, USA

L. S. Stone $\cdot$ T. K. Y. Lim

Alan Edwards Center for Research on Pain, McGill University,

Montreal, QC, Canada repetitive behaviors among individuals with neurodevelopmental disorders associated with intellectual disability.

Keywords Salivary · Self-injury · Stereotypy · HPA axis · Hypothalamic-pituitary-adrenal axis (HPA) · Sympathoadreno-medullary system (SAM)

Self-injurious behavior (SIB) is a relatively prevalent behavior disorder among populations of people with neurodevelopmental disorders associated with intellectual and developmental disabilities (IDD), with estimated prevalence rates between 5-20\% of persons with IDD engaging in SIB (Schroeder et al. 2001). The presentation of SIB can be severe, characterized by non-accidental and self-inflicted head-banging, body-hitting, eye-gouging, self-biting, hairpulling, and other activities causing injury. The pathophysiology of SIB is poorly understood, and consequentially, the disorder can be difficult to treat effectively. There are no standard first choice pharmacological treatments for SIB, making the clinician's judgement the primary determinant of treatment (Osman and Loschen 1992). Limited research on biological mechanisms and their markers has impeded the identification of specific and effective forms of psychopharmacological treatment (but see Sandman et al. 1999). The study of biological markers, therefore, may be especially useful in aiding diagnosis, treatment course decisions, and in gaining a greater understanding of SIB pathophysiology.

Studies of biological markers among individuals with SIB and IDD have demonstrated a relationship between SIB and stress, which has mostly been focused around the hypothalamic-pituitary-adrenal (HPA) axis. After a SIB episode, plasma $\beta$-endorphin levels increase and uncouple from adrenocorticotropic hormone (ACTH; Sandman et al. 1997; Sandman et al. 2003). Under normal circumstances, ACTH and $\beta$-endorphin levels are highly correlated, as they 
are both derived from the parent peptide proopiomelanocortin (Guillemin et al. 1977). In addition, individuals with IDD exhibiting SIB have also been found to have elevated saliva cortisol levels, and cortisol levels were correlated with SIB severity (Symons et al. 2003). Both the $\beta$-endorphin-ACTH uncoupling and altered cortisol levels among individuals with IDD and SIB suggest that the HPA stress axis is altered in individuals with IDD exhibiting SIB (Sandman et al. 1997; Sandman et al. 2003; Symons et al. 2003). It is unlikely that these alterations in the stress response are generalizable to all persons exhibiting SIB. For example, not all individuals have increases in $\beta$-endorphin levels after self-injury (Sandman et al. 1997; Sandman et al. 2003).

There is also a great deal of comorbidity among individuals with IDD with SIB and stereotypic motor behavior. The coexpression of stereotypy and SIB is estimated at upwards of $50 \%$ in children and adults with intellectual disability (Bodfish et al. 1995; Rojahn 1986). Epidemiological studies have shown a strong association between SIB and various stereotypic behaviors among individuals with IDD (Rojahn 1986). These findings suggest individuals who express SIB and stereotypy may fall into a different category of abnormal behavior than those with SIB only or stereotypy only. Although specific biochemical abnormalities have yet to be demonstrated among these subtypes, Hessl et al. (2008) showed genotype differences in the serotonin transporter (5-HTTLPR) for stereotypy [LL $>$ SL, SS]) in a sample of individuals with fragile $\mathrm{X}$ syndrome.

Pharmacological evidence also supports indirectly the possibility of separate subgroups of individuals with both SIB and stereotypy. For example, individuals with IDD with both stereotypy and SIB react differently to sedative-hypnotics than persons with only SIB, only stereotypy, or neither behavior disorder. In this sample, individuals with both SIB and stereotypy were most likely to respond to large doses of sedative-hypnotics by "maintaining wakefulness, and producing either resistive, combative, restless, uncooperative, or abusive behaviour" (Barron and Sandman 1983). In another study, increased opiate sensitivity in SIB skin pickers with stereotypy was found, when compared with patients with trichotillomania, a less severe form of stereotypy (Frecska and Arato 2002). Together, pharmacological and epidemiological investigations suggest that SIB in individuals with pathologic (i.e., based on Diagnostic and Statistical Manual on Mental Disorders, Fourth Edition (DSM-IV) criteria) stereotypic behavior may exist as a subgroup or possibly separate behavioral phenotype with a unique biological profile.

A separate stress system which has received almost no attention in humans in the study of stereotypy and SIB is the sympatho-adreno-medullary (SAM) system. In animal models, sympathetically dominated pigs, when tethered over long-term, develop stereotypies and increased sympathetic tone. Stereotypic behavior was found to decrease heart rate in these animals and counteract the overactive sympathetic tone (Schouten and Wiegant 1997). Heart rate monitoring in rhesus monkeys has shown that SIB monkeys experience an increase in heart rate before a bout of self-injury and that self-biting returns heart rate back to basal levels (Novak 2003). In humans, evidence for altered SAM response in SIB and stereotypy has been mostly pharmacological. Although no placebo-controlled double-blind clinical trials have been performed, a number of studies have documented efficacy of adrenergic attenuating drugs such as clonidine and propranolol in the treatment of SIB (Blew et al. 1999; Luchins and Dojka 1989; Philipsen et al. 2004; Ruedrich et al. 1990; Symons et al. 2004). These findings suggest that stereotypic behaviors and SIB may be elicited in situations of SAM over-reactivity.

Given the evidence summarized briefly above, the overall goal of our work was to extend the study of biomarkers in SIB research among individuals with IDD. We examined two specific stress-related biomarkers based, in part, on the findings from other independent research groups in areas of stress physiology relevant to the problem of SIB in individuals with IDD. Because this is a highly vulnerable population with cognitive and communicative impairments, we adopted a noninvasive approach based on collecting and assaying saliva. Salivary assessment provides a noninvasive means of examining biological aspects of and stress-related HPA/SAM activity in routine clinical settings (Hellhammer et al. 2009).

Our general strategy involved first collecting saliva on matched (gender, age, developmental level) individuals with IDD, with and without SIB, for initial comparisons. Then, within-SIB subgroup comparisons were made based on the presence/absence of stereotyped behavior. We tested the hypothesis that individuals with chronic SIB would display altered basal stress-related biochemical markers. As a marker of HPA axis function, we measured salivary cortisol levels (Stahl and Dorner 1982). We measured salivary $\alpha$-amylase enzyme activity as a marker of SAM axis function (Chatterton et al. 1996; Nater et al. 2005; Rohleder et al. 2004; van Stegeren et al. 2006).

\section{Method}

\section{Participants}

Individuals were recruited from the total residential population $(N=366)$ of a regional center for adults with intellectual and associated neurodevelopmental disorders and related disabilities. From the subgroup of residents with chart diagnoses that included self-injury $(n=98)$, a sample of 51 adults $(57 \%$ male) with intellectual and developmental disabilities participated in this study (SIB group, $n=34$; no SIB control group, $n=17$ ). Control participants were matched on chronological 
age (within a 5-year period), level of intellectual disability (exact match based on DSM-IV levels-mild, moderate, severe, or profound), and gender (exact match). The mean age was 48.4 years (range, 20-65 years) for the SIB group and 43.8 years (range, 30-65 years) for the control group. Males comprised $62 \%$ of the SIB group and $47 \%$ of the control group; $8 \%$ of the sample functioned in the moderate range of mental retardation; $10 \%$ of the sample functioned in the severe range of mental retardation, and $82 \%$ of the sample functioned in the profound range of mental retardation. Chi-square tests showed no significant differences in the demographic (age, IQ, gender) characteristics of SIB and non-SIB groups. Participants resided in a residential facility providing services for individuals with complex behavioral and/or medical needs.

Inclusion/exclusion criteria The following criteria were used to select non-SIB participants (male or female). Inclusion: (1) have a diagnosis of severe/profound or moderate intellectual disability (based on DSM-IV criteria and standard intellectual and adaptive behavioral test results within the previous 3 years as documented through existing chart review); (2) be between the ages of 18-65 years. Exclusion: (1) serious accompanying chronic health impairments that are considered to be painful (e.g., chronic reflux or otitis media as determined by subjects' physician record review and/or examination if necessary).

The following criteria were used to select SIB participants (male or female): Inclusion: (1) have a diagnosis of severe/profound or moderate intellectual disability (based on DSM-IV criteria and standard intellectual and adaptive behavioral test results within the previous 3 years as documented through existing chart review); (2) be between the ages of 18-65 years; (3) exhibits SIB either hourly or at least daily in bouts or discrete episodes (based on staff report using the Behavior Problems Inventory (Rojahn et al. 2001); (4) persistent SIB (present for at least the last 12 months; based on staff report/behavioral incidents in records); (5) receiving treatment for SIB that is currently stable (not planned on being changed in the next month); (6) parent or guardian informed consent. Exclusion: (1) serious accompanying chronic health impairments that are considered to be painful (e.g., chronic reflux or otitis as determined by subjects' physician record review and/or examination if necessary) or (2) specific syndromes known to be associated with SIB (e.g., Lesch-Nyan syndrome, Cornelia de Lange syndrome).

Saliva collection and processing

One milliliter of unstimulated saliva was collected in the early afternoon (approximately 2:00 PM $[$ mean $=1: 44 \mathrm{PM}$,
$\mathrm{SD}=36 \mathrm{~min}$ ] with one exception for one participant collected at 10:00AM: collection times did not differ significantly by group [SIB vs control]). Participants received no food, liquid, or tooth brushing nor did they display self-injurious behaviors within the hour prior to collection. Each residential living unit had in place a standard surveillance system for monitoring severe behavior disorders including SIB. Research staff were present for the $1 \mathrm{~h}$ prior to the collection time to corroborate the absence of SIB. Collection days were randomly chosen across participants (with some breakdown in this given idiosyncratic changes in individual participant's daily schedule). Saliva was collected by swabbing the individual's mouth using Toothettes. Toothettes were cut and placed into Salivette tubes and centrifuged at 3,000 rpm for $5 \mathrm{~min}$. For each individual, the resultant saliva from each Toothette was then mixed together using a Vortex for $45 \mathrm{~s}$ at a speed of 1,820 RPM, aliquoted into labeled microtubes, and stored at $-80 \mathrm{C}$ until used.

\section{Assays}

Group membership was blinded prior to conducting assays. On the day of the assay, samples were thawed and vortexed. Freeze-thaw cycles were minimized.

Cortisol Cortisol levels where measured by enzyme immunoassay using a commercially available colorimetric kit according to the manufacturer's instructions (Salimetrics, State College, PA; Catalog \# I-3012). Colorimetric determination was performed on a SpectraMax M2e plate reader (Molecular Devices, Sunnyvale, CA). The assay has a calibration range $0.12-30 \mathrm{ng} / \mathrm{ml}$ with a sensitivity of $0.03 \mathrm{ng} / \mathrm{ml}$. All measurements were performed in triplicate and averaged.

Salivary $\alpha$-amylase Salivary $\alpha$-amylase was measured using a commercially available enzyme reaction assay according to the manufacturer's instructions (Salimetrics, State College, PA; Catalog \# 1-1902). The kinetic formation of cleaved 2-chloro- $p$-nitrophenol from 2-chloro$p$-nitrophenol-linked maltotriose by salivary $\alpha$-amylase was spectrophotometrically measured at $405 \mathrm{~nm}$ and by colorimetry using a SpectraMax M2e plate reader (Molecular Devices, Sunnyvale, CA). The amount of salivary $\alpha$-amylase activity present in the sample is directly proportional to the increase in absorbance at $405 \mathrm{~nm}$. All measurements were performed in triplicate.

Analyses and statistics

A matched between-groups design was used. Descriptive statistics were used to characterize measures of central 
tendency and variability for each of the putative biomarkers across and within groups. Parametric (when appropriate) and nonparametric statistics were used to compare biomarkers across groups. Analyses were performed with and without outliers (beyond 2 SDs from the mean). Correlational and additional comparative analyses were used to examine relations between demographic, procedural, and individual characteristics and biomarkers within the SIB group.

\section{Results}

Correlations and categorical variable comparisons

A number of demographic (age, gender, intellectual disability level), procedural (time of day of collection), and SIB (reported frequency) variables were examined to determine whether they were associated with the biomarker variables. Alpha-amylase differed significantly by level of intellectual impairment across groups when tested using the Kruskal-Wallis at $\chi^{2}(2)=8.15, p=0.017$ (Table 1). Total stereotypy, when measured by the Behavior Problems Inventory (regardless of stereotypic movement disorder [SMD] diagnosis; see below), was significantly correlated with $\alpha$-amylase $(r=0.36, p<0.05)$ among all participants with SIB and stereotypy $(n=34)$. With the removal of two outliers, $\alpha$-amylase was strongly correlated with total stereotypy $(r=0.57, p<0.001$; Fig 1$)$. Cortisol and salivary $\alpha$-amylase were non-significantly negatively correlated in this sample for SIB only cases $(r=-0.22)$ and for SIB + SMD cases $(r=-0.22)$.

\section{Between-group comparisons (SIB vs no SIB)}

We compared all SIB cases with the no SIB cases. Levene's test statistics indicated borderline or significant homogeneity of variance for most groups. As such, the nonparametric Mann-Whitney $U$ test was employed to test differences for

Table 1 Descriptive and Kruskal-Wallis results for level of intellectual impairment subgroups

\begin{tabular}{llllll}
\hline Variable & Moderate & Severe & Profound & $\chi^{2}(\mathrm{df})$ & $\begin{array}{l}p \\
\text { Value }\end{array}$ \\
\hline$\alpha$-Amylase & & & & $8.15(2)$ & 0.017 \\
$n$ & 4 & 5 & 42 & & \\
$\mathrm{M}(\mathrm{SD})$ & $32.8(22.8)$ & $166.1(99.5)$ & $117.9(97.2)$ & & \\
$\mathrm{C}_{\mathrm{V}}$ & 1.44 & 1.67 & 1.21 & & \\
$\mathrm{Cortisol}$ & & & & $2.49(2)$ & 0.288 \\
$n$ & 4 & 5 & 42 & & \\
$\mathrm{M}(\mathrm{SD})$ & $18.5(32.5)$ & $1.3(1.6)$ & $3.17(9.8)$ & & \\
$\mathrm{C}_{\mathrm{V}}$ & 0.57 & 0.81 & 0.32 & & \\
\hline
\end{tabular}

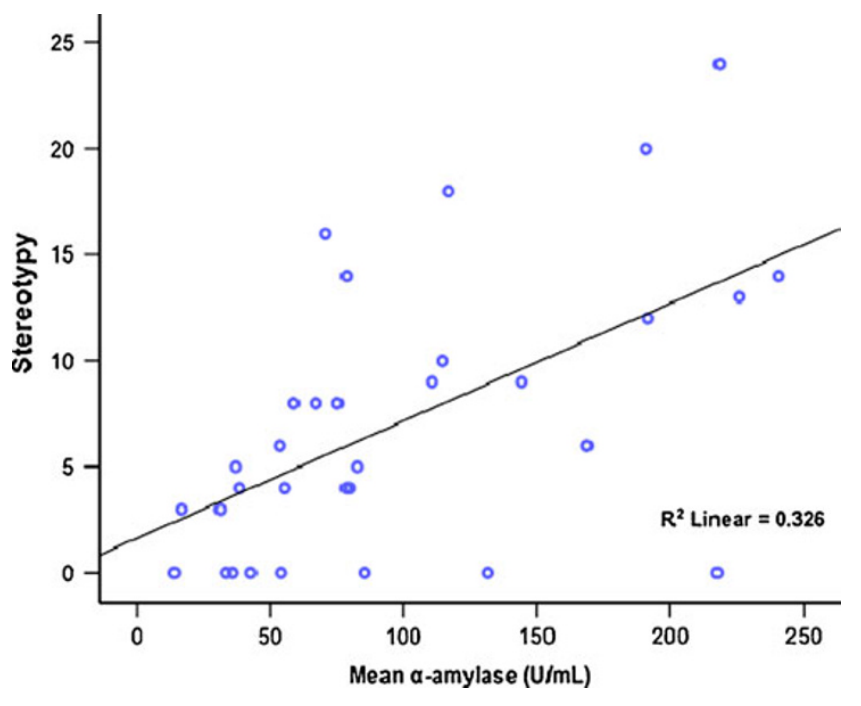

Fig. 1 Scatter plot of salivary $\alpha$-amylase and total stereotypy based on the Behavior Problems Inventory among participants with SIB $(n=32, r=0.571, p<0.001)$

salivary concentrations of $\alpha$-amylase and cortisol. Descriptive and Mann-Whitney $U$ test statistics and results are presented in Table 2. Significant differences between groups (SIB vs. no SIB) were detected for cortisol.

Between-group comparisons (SIB subgroups)

$S I B$ vs SIB + SMD vs control Initial data analysis indicated adequate normality and homogeneity (using Levene's test) for about half of the variables for the comparison of the biomarkers across a SIB-only group, a SIB group that met $D S M-I V$ diagnostic criteria for SMD and the no SIB controls. Analysis of variance was employed to test differences for salivary concentrations of each biomarker across these three groups. No significant group differences were detected for this parametric comparison. Because homogeneity was borderline for the biomarkers $(\sim 50 \%)$, the nonparametric Kruskal-Wallis test was employed. Results of this test are presented in Table 3. Significant differences across groups were indicated for $\alpha$-amylase and cortisol. A further comparison was made specific to individuals with SIB comparing SIB only and SIB + SMD. A significant difference was detected between groups for the biomarker $\alpha$-amylase with $U=69, z=-2.59, p=0.009$.

\section{Discussion}

SIB among adults with severe IDD represents a serious problem occurring in up to $20 \%$ of system-wide surveys and clinical samples. Very little is known about the pathophysiological mechanisms underlying SIB; a long- 
Table 2 Descriptive and Mann-Whitney $U$ test results for SIB and no SIB subgroups

\begin{tabular}{llllll}
\hline Variable & SIB & No SIB & $Z$ score & U & $p$ Value \\
\hline$\alpha$-Amylase & & & -0.66 & 319 & .510 \\
$n$ & 34 & 17 & & & \\
M (SD) & $110.1(79.3)$ & $127.9(126.9)$ & & & \\
Cortisol & & & -2.79 & 196 & 0.005 \\
$n$ & 34 & 17 & & & \\
$\mathrm{M}(\mathrm{SD})$ & $5.3(15.1)$ & $1.9(5.1)$ & & & \\
\hline
\end{tabular}

standing but little directly tested assumption is that stress and arousal play a role in the expression of the behavior. Limited work suggests an association between SIB and the HPA axis, but findings are mixed in this regard (Kemp et al. 2008; Sandman et al. 1990; Symons et al. 2003; Verhoeven et al. 1999). No work has specifically examined the SAM system although there is an apparent association between this system and SIB/stereotypy in non-human primate research as well as pharmacological work specific to adrenergic medications (Blew et al. 1999; Novak 2003; Philipsen et al. 2004; Symons et al. 2004; Tiefenbacher et al. 2000). The goal of this preliminary study was to noninvasively examine the HPA axis and SAM systems by testing two salivary biomarkers for each system, cortisol and $\alpha$-amylase, respectively.

Overall, individuals with SIB had elevated levels of cortisol compared with matched individuals (age, gender, level of intellectual impairment) who did not self-injure. Our current results replicate previous findings of elevated cortisol among those with chronic SIB (Symons et al. 2003; Verhoeven et al. 1999). Among the SIB group, there were significant differences in salivary $\alpha$-amylase levels between individuals with and without a co-occurring SMD. The observed elevated levels of $\alpha$-amylase associated with

Table 3 Descriptive and Kruskal-Wallis results for SIB, SIB+SMD, and control subgroups

\begin{tabular}{llllll}
\hline Variable & SIB & SIB+SMD & Control & $\chi^{2}(\mathrm{df})$ & $\begin{array}{l}p \\
\text { Value }\end{array}$ \\
\hline$\alpha$ - & & & & $7.68(2)$ & 0.021 \\
\multicolumn{1}{c}{ Amylase } & & & & & \\
$\mathrm{M}(\mathrm{SD})$ & $88.1(87.4)$ & $134.7(62.8)$ & $127.9(126.9)$ & & \\
$\mathrm{C}_{\mathrm{V}}$ & 1.01 & 2.14 & 1.01 & & \\
$\mathrm{Cortisol}$ & & & & $6.39(2)$ & 0.041 \\
$n$ & 18 & 16 & 17 & & \\
$\mathrm{M}(\mathrm{SD})$ & $5.7(16.1)$ & $5.0(14.4)$ & $1.88(5.12)$ & & \\
$\mathrm{C}_{\mathrm{V}}$ & 0.35 & 0.35 & 0.37 & & \\
\hline
\end{tabular}

chronic SIB and stereotyped behavior as well as a positive correlation with severity of IDD are novel findings and may indicate altered sympathetic activity. Given that the findings were specific to a subgroup of individuals with SIB, it suggests that future work should attend to co-morbid conditions (e.g., stereotypy) occurring among individuals with SIB. Frecska and Arato (2002) showed that individuals with SMD and SIB in the form of skin-picking differed significantly on an opioid sensitivity test than a comparable group with less serious forms of SIB (hair-pulling). At the risk of oversimplifying a complex disorder, such subgroup differences logically support different therapeutic approaches, although a clinical trial would be needed to definitively test this logic.

There are several study-specific issues that limit the generality of the study findings reported here. The sample was not formed randomly, and therefore, the results are specific to the individuals reported on herein. The sample was residential and composed primarily of individuals with significant intellectual disabilities, and therefore, it is not clear how our findings would generalize to a more heterogeneous and community-based intellectual disability population. We did not directly measure environmental variables (e.g., social density, staff interactions, etc.), and so their possible effects are unknown, but future work may be designed to consider their influence on the systems underlying the two biomarkers we studied (cortisol, sAA). We did, however, try to be clear about the sample formation and composition to facilitate logical generalization to comparable individuals with IDD. Although we were careful to collect saliva from individuals under as standardized conditions as possible with respect to time of day and environmental events, factors of timing (in relation to behavioral events) and decay may impact the usefulness and measurement validity of saliva collection (see Hellhammer et al. 2009 and Rohleder and Nater 2009, for excellent reviews regarding methodological considerations concerning saliva). Also, the single time-point for collection, although common to all participants (thereby controlling for time of day) provides a very limited window into the activity of either system (HPA, SAM).

Given the limitations described above, we can only speculate on what the differences in $\alpha$-amylase means for the SAM axis in these groups. It may be that there is elevated sympathetic tone in people with both SIB and stereotypy. Individuals with SIB plus SMD may respond to stress and arousal in different ways than individuals with SIB only. Among real-world tests of acute stress effects in non-disabled study samples, stress-mediated (elevated) total protein and $\alpha$-amylase were found to be significantly higher immediately preceding a stressful event (academic test) than during non-stress times ( 2 and 6 weeks later) among dental students (Bosch et al. 1996). In a single-case 
psychiatric report of an adult woman diagnosed with borderline personality disorder, Sachsse et al. (2002) documented increasing nocturnal urinary cortisol levels were associated with self-mutilation and that urinary cortisol levels dropped following episode of SIB. Their discussion focused on the possible relation between stress and affect regulation in relation to self-injury. Whether similar findings related to acute stressors or in relation to acts of SIB would have similar effects among individuals with IDD is an open question. Our results do provide one possible mechanism for how clonidine or propranolol would work to reduce SIB (Blew et al. 1999; Luchins and Dojka 1989; Ruedrich et al. 1990; Symons et al. 2004). There is the possibility that the SIB +SMD group would be more reactive to propranolol/clonidine which, in turn, raises the possibility that amylase could be used as a marker to screen individuals who may be responsive to propranolol/ clonidine treatment.

In this work, we demonstrated biochemical differences between control, SIB, and SIB +SMD groups, observable in the saliva of persons with intellectual impairments associated with neurodevelopmental disorders, suggesting it may be possible to use biomarkers to catalog and discriminate different behavioral disorders. Although the ultimate functional significance of bioactive peptides found in the human major salivary glands remains an area of active research, it is clear that chronic stress and related environmental effects produce profound changes in various autonomic nervous system functions both in animal models and in human subjects (Hellhammer et al. 2009; Nater and Rohleder 2009; Rohleder et al. 2004). It is therefore possible that these changes are reproduced in many parts of the autonomic nervous system, including those supplying human salivary glands. Thus, the characteristic pattern of activity should be reflected in changes in the concentration ratios of selected neuropeptides in the saliva.

To this point-characteristic patterns of activity-the work reported here raises additional issues specific to assumptions underlying our approach (and much of the conventional wisdom) to biomarker work among vulnerable clinical populations with compromised intellectual and communicative abilities. If the level of a putative stress biomarker is elevated, this is taken as evidence of elevated stress or stress-related activation. Absent an independent and specific stress test, such conclusions are rarely warranted. Raised levels of cortisol, for example, may reflect down-regulated glucocorticoid receptors (GR). A key difference may be whether the effects are related to acute or chronic activation-different mechanisms may become more or less relevant (for example, the relative degree of interplay between corticotrophin-releasing factor neurons, adrenocorticotrophic hormone (ACTH), and sympathetic activation contribute considerable covariation in salivary cortisol levels). More generally, the notion of inferring simple linear effects ('high cortisol' $=$ high stress) is not tenable - salivary cortisol levels vary considerably with respect to adrenal capacity, liver metabolism, cortisol binding globuline, and GR gene variants (Hellhammer et al. 2009). Given our preliminary observations about differences in two stress-related markers, there are two specific areas that warrant additional attention, the first is our understanding of whether different subgroups with SIB and stereotypy represent different pathways to a disorder and the second is our understanding of stress and sympathetic/parasympathetic response and regulation.

With respect to the former-different subgroups-motor stereotypy has been associated with striatal morphology and function, with striatal dopamine in particular positively associated with elevated stereotypy (Aliane et al. 2011; Hollander et al. 2005). Frontal-striatal dopamine pathways have likewise been implicated in the learning and maintenance of repetitive behaviors in general (Graybiel 2008). Lower sAA among the SIB group may indicate reduced output of norepinepherine, suggesting diminished upstream dopaminergic function. It may be that motor stereotypy is distinct from repetitive self-injury with regard to its neural underpinnings. For those with both SIB and SMD, stereotypy may have a moderating influence on striatal dopamine and ACTH function. Dampened sAA among those with SIB alone may reflect circuit-level changes resulting from prolonged dopamine release associated with self-injury, consistent with an addiction model of SIB (Graybiels 2008; Sandman et al. 1999). Hypodopaminergic function within the striatum has been previously associated with self-injury among persons with Lesch-Nyhan syndrome (Wong et al. 1996). Alternatively, the findings may reflect distinct SIB pathogenesis with regard to neurocircuitry. What both SIB groups (those with and without SMD) share in common was elevated cortisol, which indicates higher basal arousal relative to controls. While it is possible that stress and stress responsivity play a role in the production of repetitive behavior, it is difficult to discern the direction of the stress-behavior relationship with biomarker data alone (Major et al. 2009).

With regard to the last point made above-stress response/ regulation - the general approach has been to interpret elevated markers in the framework of normal physiologic response to stress (see Moghaddam 2002 for an extended discussion). It may be, however, that the stress response itself is abnormal and that we have yet to work out how the vulnerabilities created by the different neural insults that ultimately lead to intellectual disability regulate the different neural stress axes. In other words, we are not yet well positioned to know whether our observations reflect normal or abnormal stress physiology (i.e., the system is working in overdrive vs the system is working differently). 
To conclude, our results suggest that, for a vulnerable population, collection of salivary samples is a practical and noninvasive means of obtaining data pertaining to biomarkers for HPA axis (cortisol) and SAM system activity ( $\alpha$-amylase) among individuals with significant IDD. The observed elevated levels of $\alpha$-amylase associated with chronic SIB and stereotyped behavior as well as a positive correlation with severity of IDD are novel findings and may indicate altered sympathetic activity. These preliminary findings warrant further exploration into the role of the SAM system in the pathophysiology of SIB and related repetitive behaviors among individuals with intellectual disabilities and neurodevelopmental disorders.

\section{References}

Aliane V, Pérez S, Bohren Y, Deniau JM, Kemel ML. Key role of striatal cholinergic interneurons in processes leading to arrest of motor stereotypies. Brain. 2011;134:110-8.

Barron J, Sandman CA. Relationship of sedative-hypnotic response to self-injurious behavior and stereotypy by mentally retarded clients. Am J Ment Defic. 1983;88:177-86.

Blew P, Luiselli JK, Thibadeau S. Beneficial effects of clonidine on severe self-injurious behavior in a 9-year-old girl with pervasive developmental disorder. J Child Adolesc Psychopharmacol. 1999;9:285-91.

Bodfish JW, Crawford TW, Powell SB, Parker DE, Golden RN, Lewis $\mathrm{MH}$. Compulsions in adults with mental retardation: prevalence, phenomenology, and comorbidity with stereotypy and self-injury. Am J Ment Retard. 1995;100:183-92.

Bosch JA, Brand HS, Ligtenberg TJ, Bermond B, Hoogstraten J, Nieuw Amerongen AV. Psychological stress as a determinant of protein levels and salivary-induced aggregation of Streptococcus gordonii in human whole saliva. Psychosom Med. 1996;58:374 82 .

Chatterton RT, Vogelsong KM, Lu YC, Ellman AB, Hudgens GA. Salivary alpha-amylase as a measure of endogenous adrenergic activity. Clin Physiol. 1996;16:433-48.

Frecska E, Arato M. Opiate sensitivity test in patients with stereotypic movement disorder and trichotillomania. Prog Neuro-psycho pharmacol Biol Psychiatry. 2002;26:909-12.

Guillemin R, Vargo T, Rossier J, Minick S, Ling N, Rivier C, et al. beta-Endorphin and adrenocorticotropin are selected concomitantly by the pituitary gland. Science. 1977;197:1367-9.

Graybiel AM. Habits, rituals, and the evaluative brain. Annu Rev Neurosci. 2008;31:359-87.

Hellhammer DH, Wust S, Kudielka BM. Salivary cortisol as a biomarker in stress research. Psychoneuroendocrinology. 2009; $34: 163-71$

Hessl D, Tassone F, Cordeiro L, Koldewyn K, McCormick C, Green $\mathrm{C}$, et al. Aggression and stereotypic behavior in males with fragile X syndrome- moderating secondary genes in a "single gene" disorder. J Autism Dev Disord. 2008;38:184-9.

Hollander E, Anagnostou E, Chaplin W, Esposito K, Haznedar MM, Licalzi $\mathrm{E}$, et al. Striatal volume on magnetic resonance imaging and repetitive behaviors in autism. Biol Psychiatry. 2005;58:226-32.

Kemp AS, Fillmore PT, Lenjavi MR, Lyon M, Chicz-DeMet A, Touchette PE, et al. Temporal patterns of self-injurious behavior correlate with stress hormone levels in the developmentally disabled. Psychiatr Res. 2008;157:181-9.
Luchins DJ, Dojka D. Lithium and propranolol in aggression and selfinjurious behavior in the mentally retarded. Psychopharmacol Bull. 1989;25:372-5.

Major CA, Kelly BJ, Novak MA, Davenport MD, Stonemetz KM, Meyer JS. The anxiogenic drug FG7142 increases self-injurious behavior in male rhesus monkeys (Macaca mulatta). Life Sci. 2009;85:753-8.

Moghaddam B. Stress activation of glutamate neurotransmission in the prefrontal cortex: Implications for dopamine-associated psychiatric disorders. Biol Psychiatry. 2002;51:775-87.

Nater UM, Rohleder N. Salivary $\alpha$-amylase as a non-invasive biomarker for the sympathetic nervous system: Current state of research. Psychoneuroendocrinology. 2009;34:486-96.

Nater UM, Rohleder N, Gaab J, Berger S, Jud A, Kirschbaum C, et al. Human salivary alpha-amylase reactivity in a psychosocial stress paradigm. Int J Psychophysiol. 2005;55:333-42.

Novak MA. Self-injurious behavior in rhesus monkeys: new insights into its etiology, physiology, and treatment. Am J Primatol. 2003;59:3-19.

Osman OT, Loschen EL. Self-injurious behavior in the developmentally disabled: pharmacological treatment. Psychopharmacol Bull. 1992;28:439-49.

Philipsen A, Richter H, Schmahl C, Peters J, Rusch N, Bohus M, et al. Clonidine in acute aversive inner tension and self-injurious behavior in female patients with borderline personality disorder. J Clin Psychiatry. 2004;65:1414-9.

Rohleder N, Nater UM. Determinants of salivary $\alpha$-amylase in humans and methodological considerations. Psychoneuroendocrinology. 2009;34:469-85.

Rohleder N, Nater UM, Wolf JM, Ehlert U, Kirschbaum C. Psychosocial stress-induced activation of salivary alphaamylase: an indicator of sympathetic activity? Ann NY Acad Sci. 2004;1032:258-63.

Rojahn J. Self-injurious and stereotypic behavior of noninstitutionalized mentally retarded people: prevalence and classification. Am J Ment Defic. 1986;91:268-76.

Rojahn J, Matson JL, Lott D, Esbensen A, Smalls Y. The Behavior Problems Inventory: An instrument for the assessment of selfinjury, stereotyped behavior, and aggression/destruction in individuals with developmental disabilities. J Autism Dev Disord. 2001;31:577-88.

Ruedrich SL, Grush L, Wilson J. Beta adrenergic blocking medications for aggressive or self-injurious mentally retarded persons. Am J Ment Retard. 1990;95:110-9.

Sachsse U, Von der Heyde S, Huether G. Stress regulation and selfmutilation. Am J Psychiatry. 2002;159:672.

Sandman CA, Barron JL, Chicz-DeMet A, DeMet EM. Plasma B-endorphin levels in patients with self-injurious behavior and stereotypy. Am J Ment Retard. 1990;95:84-92.

Sandman CA, Hetrick W, Taylor DV, Chicz-DeMet A. Dissociation of POMC peptides after self-injury predicts responses to centrally acting opiate blockers. Am J Ment Retard. 1997;102:182-99.

Sandman CA, Spence MA, Smith M. Proopiomelanocortin (POMC) disregulation and response to opiate blockers. Ment Retard Dev Disabil Res Rev. 1999;5:314-21.

Sandman CA, Touchette P, Lenjavi M, Marion S, Chicz-DeMet A. betaEndorphin and ACTH are dissociated after self-injury in adults with developmental disabilities. Am J Ment Retard. 2003;108:414-24.

Schouten WG, Wiegant VM. Individual responses to acute and chronic stress in pigs. Acta Physiol Scand Suppl. 1997;640:88-91.

Schroeder SR, Oster-Granite ML, Berkson G, Bodfish JW, Breese GR, Cataldo MF, et al. Self-injurious behavior: Gene-brainbehavior relationships. Ment Retard Dev Disabil Res Rev. 2001;7:3-12.

Stahl F, Dorner G. Responses of salivary cortisol levels to stress situations. Endokrinologie. 1982;80:158-62. 
Symons FJ, Sutton KA, Walker C, Bodfish JW. Altered diurnal patterns of salivary substance $\mathrm{P}$ in adults with developmental disabilities and chronic self-injury. Am J Ment Retard. 2003;108:13-8.

Symons FJ, Thompson A, Realmuto G. Clonidine for self-injurious behavior. J Am Acad Child Adolesc Psychiatry. 2004;43:1324-5.

Tiefenbacher S, Novak MA, Jorgensen MJ, Meyer JS. Physiological correlates of self-injurious behavior in captive, socially-reared rhesus monkeys. Psychoneuroendocrinology. 2000;25:799817. van Stegeren A, Rohleder N, Eveaerd W, Wolf OT. Salivary alpha amylase as marker for adrenergic activity during stress: effect of betablockade. Psychoneuroendocrinology. 2006;31:137-41.

Verhoeven WM, Tuinier S, van den Berg YWMM, Coppus AMW, Fekkes D, Pepplinkhuizen L, et al. Stress and self-injurious behavior; hormonal and serotonergic parameters in mentally retarded subjects. Pharmacopsychiatry. 1999;32:13-20.

Wong DF, Harris JC, Naidu S, Yokoi F, Marenco S, Dannals RF, et al. Dopamine transporters are markedly reduced in Lesch-Nyhan disease in-vivo. PNAS. 1996;93:5539-43. 